\title{
Présence du traducteur dans le processus de traduction spécialisée
}

\author{
The presence of a translator in the process \\ of translating specialized texts
}

\author{
Teresa Tomaszkiewicz \\ Uniwersytet im. Adama Mickiewicza, Poznań \\ tomaszki@amu.edu.pl
}

\begin{abstract}
This article deals with the issue of the role of a translator in translating specialized texts. It is theoretically assumed that a translator of that kind of texts is merely a transferor of information and should not intervene with the meaning of the given text. Numerous studies show, however, that a translator of specialized texts - subject to the nature of the given text - must employ a whole range of translation techniques which aim at explicating certain concealed or unknown messages being conveyed to the receiving party. In this article we strive to prove that these explanatory techniques can take on a totally different dimension when applied to scientific or pragmatic texts. Therefore it can be inferred that translating scientific texts provides for different interventions on the part of the translator than in the case of translating utilitarian texts. Nevertheless, each such intervention is to some extent creative from the point of view of the role of the translator in the process of the transfer.
\end{abstract}

Keywords: specialized translation, scientific texts, utilitarian texts, translator's traces, explanatory techniques

\section{INTRODUCTION}

La traduction est pratiquée depuis que les gens parlent des langues différentes tandis que la traductologie en tant que science a fait ses premiers pas dans les années cinquante du $\mathrm{XX}^{\mathrm{e}}$ siècle pour n'être reconnue dans les universités comme une science indépendante qu'il y a 25 ans. Or, comme le disait Ballard en 1995 «la perception d'un tel changement implique qu'existait un état antérieur fait de silence, d'ignorance et souvent aussi de mépris. On connaît l'image du traducteur honteux, toujours accusé de tous les maux ; quant à la traduction, elle n'était guère digne d'étude et on méconnaissait sa place dans 
l'histoire de la transmission des savoirs et des cultures » (Ballard, 1995 : couverture). Et pourtant, le travail des traducteurs, qui ont toujours été présents dans l'histoire de l'humanité, a considérablement marqué les relations entre les peuples et les cultures. Or, le statut social de ces médiateurs de communication humaine a été perçu différemment à travers les époques.

L'histoire de la traduction (...) révèle une multiplicité de circonstances et, par conséquent, de modes de traduction. Parmi ces circonstances, il faut entendre d'abord le traducteur, son contexte sociohistorique, ses motivations, ses objectifs, ses limites et ses commanditaires éventuels. Ensuite, les textes, leur fonction originale, leurs destinataires, leur époque, leur nature littéraire, religieuse, scientifique, technique ou juridique. Tout cela a fait jouer aux traducteurs un rôle dans l'histoire de l'humanité - rôle qui, malgré tout, reste généralement dans l'ombre encore aujourd'hui (Bastin et Cormier, 2007 : 21).

Voilà donc la vision d'un traducteur "dans l'ombre », un traducteur perçu comme " une vitre transparente », quelqu'un qui ne peut pas ou même ne doit pas intervenir dans le contenu du texte à traduire. Cette compréhension du rôle de traducteur dans le processus de communication internationale a considérablement changé ces dernières années. Contrairement aux visions précédentes, de nouvelles approches placent le traducteur au centre du contexte communicatif international en lui attribuant la fonction de médiateur entre les textes et les contextes de leur production.

Dans cet article nous voulons nous concentrer sur le rôle du traducteur des textes spécialisés pendant la transmission des informations, en présentant les différentes formes de sa présence et de ses traces dans le texte d'arrivée. Pour le faire nous allons tout d'abord rappeler l'évolution de l'approche scientifique de la personne du traducteur, pour nous pencher avant tout sur le traducteur des textes en langues de spécialité. La définition de la langue de spécialité n'étant pas toujours admise à l'unanimité, nous allons revenir sur certains débats concernant ce phénomène pour passer ensuite à la présentation de différents types de textes spécialisés. C'est dans ce type de textes que nous pensons détecter les traces du traducteur tout en analysant leur portée et leurs fonctions. Ces fonctions souvent résultent de la nature du texte, c'est pourquoi nous pensons illustrer notre propos par deux types de textes, dont la nature est différente, à savoir un texte scientifique de psychologie et un texte pragmatique dans le domaine automobile ${ }^{1}$. Ceci nous permettra de démontrer les différentes formes d'interventions du traducteur en fonction du type de texte et le rôle qu'il joue dans le processus de transmission des messages.

\footnotetext{
${ }^{1}$ La bibliographie du corpus est présentée à la fin de cet article.
} 


\section{1. ÉVOLUTION DU RÔLE DU TRADUCTEUR DANS LA RECHERCHE}

Comme nous avons mentionné plus haut, les débuts de la traductologie remontent aux années cinquante du $\mathrm{XX}^{\mathrm{e}}$ siècle. À l'époque cette nouvelle science s'inspirait largement des découvertes de la linguistique, notamment du structuralisme. L'objet de la recherche était le transfert du sens d'un texte à un autre à la base d'une équivalence linguistique ${ }^{2}$. Dans cette conception le traducteur devait rester invisible. Personne ne réfléchissait sur ses stratégies ou ses décisions. Il devait jouer le rôle de la fameuse « vitre transparente».

A partir des années soixante-dix la sociolinguistique, la théorie de la communication et l'analyse du discours ont influencé la réflexion traductologique en mettant l'accent sur le destinataire de la traduction (théorie du skopos) et sur l'équivalence de communication ${ }^{3}$. Parmi les différents acteurs du processus on énumère évidemment le traducteur qui devient plus présent dans la recherche (p.ex. les écrits de Nord), mais il n'y occupe pas le point central.

Un tournant important s'est produit dès le début des années quatre-vingt-dix. La personne du traducteur, le contexte de son travail, ses opérations mentales (mesurées par TAP's), ses décisions traductologiques, son rôle central dans la médiation internationale deviennent d'intérêt majeur pour la recherche. La traductologie puise dans les expériences de l'analyse conversationnelle, de la sémiologie, des études sur les langues et les communications, de la théorie de l'information et bien d'autres. Depuis plusieurs années la traduction spécialisée fait aussi l'objet de recherches bien que la traduction littéraire occupe encore une place importante et même dominante dans les investigations scientifiques. Un peu à tort car la traduction littéraire ne représente aujourd'hui qu'un petit pourcentage de toutes les traductions réalisées quotidiennement dans le monde entier. On pourrait penser que la traduction littéraire donne au traducteur plus de liberté, en lui permettant de mettre en œuvre sa créativité, tandis que la traduction spécialisée reste enfermée dans la rigidité de la langue technique se caractérisant par une certaine terminologie et des structures réduites.

Or, les usages montrent que ce n'est pas la vérité et que le traducteur de spécialité doit souvent prendre les décisions qui marquent, en quelque sorte, sa présence dans le texte. Avant de le démontrer, arrêtons-nous sur la notion de texte de spécialité.

\footnotetext{
${ }^{2}$ A ce propos on peut se référer, par exemple, à des travaux de Mounin, Vinay et Darbelnet, Catford, Nida et Taber.

${ }^{3}$ Cette fois-ci citons, par exemple, les multiples travaux de Pergnier, Delisle, Selescovitch et Lederer.
} 


\section{TEXTES DE SPÉCIALITÉ}

La notion de langues de spécialité n'est pas définie d'une manière univoque. Tout le monde est d'accord, au moins intuitivement, que ces variantes linguistiques diffèrent de la langue générale, mais il est difficile de trancher net entre ce qui relève de cette spécialité et ce qui appartient encore à la langue standard. Peut-on parler des langues de spécialité au pluriel ou faut-il admettre que cette notion embrasse un même phénomène qui se manifeste sous la forme de plusieurs variantes? C'est une question qui revient constamment dans les différents débats concernant la traduction spécialisée.

Rappelons brièvement quelques positions (définitions) plus anciennes, mais plutôt classiques ressortissants de ce débat. Tout d'abord il faut tracer une première ligne de partage entre ceux qui optent pour voir dans cette variante linguistique un système qui diffère de la langue standard et ceux qui l'intègrent dans tout le système. Commençons par le premier groupe. Quemada (1978), par exemple, ramène la «langue technique » au vocabulaire. De sa part Varantola (1986) dit que les langues de spécialité sont des systèmes sémiotiques semiautonomes, basés sur la langue générale. Leur emploi suppose une éducation spéciale des utilisateurs et se limite à la communication entre les spécialistes.

Beaucoup plus nombreuses sont les opinions voyant dans les langues de spécialité un élément parmi d'autres du système linguistique en question. Par exemple, Sager, Dungworth et McDonald (1980 : 17) constatent généralement que «La différence entre les langues de spécialité et la langue générale est une différence de degré et non pas de nature. Il faut juger à quel degré les traits fondamentaux de la langue générale se retrouvent dans la langue de spécialité. L'utilisation de ces langues est déterminée par la situation de communication. Il en résulte que les utilisateurs s'en servent d'une manière plus consciente et plus précise ». De sa part Cabré (1998:112), constate que l'hétérogénéité du système linguistique ne se limite pas aux différents niveaux de la description linguistique (...) mais se manifeste aussi grâce à l'existence de ses variantes dialectales et fonctionnelles. D'après Cabré (1998) chacun de ces dialectes et de styles constitue une certaine forme de la langue de spécialité largement comprise. Kocourek (1991 : 12) qui s'est généralement occupé de la terminologie technique, a présenté une attitude purement linguistique, en constatant que la langue de spécialité est une sorte de sub-langage naturel enrichi par les éléments idéographiques et les brachygraphies qui s'intègrent à la langue générale tout en se soumettant aux règles syntaxiques de celle-ci. Toutes ces opinions sont bien résumées par Lerat (1997: 2) « aucune théorie linguistique, quelle qu'elle soit, n'a jamais isolé le fonctionnement des langues spécialisées de celui des langues naturelles en général ». 
A ces points de vue plutôt linguistiques de la définition des langues de spécialité s'oppose en quelque sorte une approche discursive. Frandsen (1998) constate, par exemple, que la notion de la langue de spécialité n'est pas opposée à la langue générale. Elle ne traduit pas un écart à cette dernière ni ne désigne sa partie. Elle permet d'étudier l'emploi d'une langue dans une situation de communication précise et s'approche ainsi de la notion de discours.

Charnock (1999: 2) propose d'établir une distinction entre la notion de langue de spécialité, définie en fonction de la discipline concernée, et celle de langage technique, présentée comme un mode d'expression, susceptible de définition linguistique indépendamment du sujet traité.

Elle admet qu'éventuellement il est possible de reconnaître les langues de spécialité par la présence d'un vocabulaire particulier et par les unités terminologiques, mais ce n'est pas un critère opérant pour plusieurs raisons.

- Il existe des termes spécialisés, en rapport avec des concepts spécifiques, pour chaque domaine de l'activité humaine, y compris des activités qui n'ont rien de technique, par conséquent tout discours devrait alors être qualifié de spécialisé.

- Il n'existe pas de définition linguistique permettant de distinguer les « unités technologiques » des unités lexicales de la langue ordinaire, à la différence des «termes savants», reconnus par leur statut étymologique particulier (cf. Mortureux, 1995).

- Les termes techniques ne sont associés à aucun critère morphologique ou lexicologique spécifique.

- En outre, de nombreux termes, réservés au départ aux seuls initiés, peuvent passer dans la langue courante au bout d'un laps de temps assez court. (Charnock, 1999)

Si l'on accepte alors que le critère terminologique n'est pas suffisant pour définir les langues de spécialité, Charnock (1999: 4) propose de puiser dans la distinction classique entre la phrase et l'énoncé en admettant, par exemple, que certains textes (techniques) énoncent des vérités génériques, de manière impersonnelle, dans un but communicatif clair et précis, et de façon aussi explicite que possible ; le rôle du contexte est alors réduit au minimum. D'autres textes (non techniques) s'expriment à un niveau plus personnel, par sousentendus, ou en évoquant des références culturelles. Ainsi la compréhension de ces textes exige une connaissance détaillée du contexte et de la situation extralinguistique.

Walkiewicz, de sa part, en traitant le problème de la traduction des textes de spécialité, s'éloigne de l'opposition phrase/énoncé en inscrivant ce processus dans la circulation des discours au sein de la société productrice et de la société réceptrice. Elle remarque que les décisions du traducteur doivent résulter de son 
jugement des situations de communication source et cible. Il s'agit de la comparaison du fonctionnement des discours en question dans les deux sociétés en contact, mais aussi de différentes relations intertextuelles au sein de chaque société en cause (Walkiewicz, $2013: 63$ ).

Il faut donc souligner que ces approches textuelle et discursive mettent en valeur justement la personne du traducteur qui devient responsable du transfert en jugeant lui-même la possibilité de compréhension du récepteur cible, la situation de communication, le contexte largement compris : thèmes abordés, intention de communication, comparaison entre le contexte source et le contexte cible. Ce n'est donc pas le privilège du traducteur littéraire de laisser ses traces dans la traduction. Le traducteur des textes spécialisés au même niveau doit prendre des décisions stratégiques pour rapprocher le texte source du récepteur. Il faut donc s'interroger sur les manifestations de ces traces du traducteur qui peuvent définir son rôle dans la traduction de textes spécialisés.

\section{TRACES DU TRADUCTEUR}

Dąmbska-Prokop $(1997,2000)$ en s'occupant justement des traces du traducteur s'inspire de l'article de Lüdi (1987) et explique que ces traces résultent de différents rôles que le traducteur joue pendant le processus de traduction. Principalement il est d'une part, le récepteur de l'original et de l'autre, le « producteur » du texte d'arrivée dont il doit prendre la responsabilité. DąmbskaProkop (2000 : 227) distingue deux types de traces : celles liées à une recherche consciente des équivalences et celles autonomes, qu'on peut remarquer sans se référer à l'original. Kaufman (2009: 139) constate que justement ces traces autonomes, par exemple, sous forme de notes du traducteur, constituent un corpus idéal pour étudier le rôle du traducteur, son attitude par rapport à sa traduction, au texte de l'original, au récepteur virtuel ou au thème du texte. Le même auteur souligne que le type et l'ampleur de ces traces résulte certainement du type de texte soumis à la traduction. Dąmbska-Prokop (1997) présente ses analyses en se référant aux textes littéraires où, semble-t-il, les interventions du traducteur seraient plus visibles, plus poussées et elles auraient un caractère créateur (cf. p.ex. Barańczak, 1992).

Par contre, les textes spécialisés que nous avons présentés plus haut ${ }^{4}$, servent principalement à la communication scientifique et technique où l'échange d'informations constitue un élément fondamental. Les auteurs de ces textes sont majoritairement des spécialistes dans un domaine concret tandis que parmi les

\footnotetext{
${ }^{4}$ Consulter la bibliographie du corpus à la fin de cet article.
} 
récepteurs on peut compter des spécialistes mais aussi d'autres personnes intéressées par la problématique en question. Le traducteur n'étant pas forcément le destinataire du texte en question en est le premier récepteur. Il en résulte qu'au niveau de la compréhension il doit tout d'abord juger comment ce texte entre en relations intertextuelles au sein de la société source et de quelle manière la traduction de ce texte peut s'intégrer au fonctionnement inter discursif cible (cf. à ce propos Walkiewicz, 2013). Ayant découvert les différences à ce niveau il peut intervenir d'une manière consciente et autonome pour neutraliser les différences possibles. Le but principal de ces interventions est d'assurer une bonne compréhension par les récepteurs cible. Or, il se peut que les caractéristiques des récepteurs source et des récepteurs cible ne soient pas les mêmes. Dans ce cas les interventions du traducteur apparaissent plus ou moins importantes. Dans la suite nous allons en présenter quelques-unes, tirées d'un texte scientifique et d'un texte de vulgarisation scientifique ${ }^{5}$, pour montrer deux façons un peu différentes de faire.

\subsection{TRACES DANS LE PARATEXTE}

Comme nous avons dit plus haut, les traces « visibles » du traducteur apparaissent le plus souvent dans les paratextes, comme les notes du traducteur ou les préfaces. Or, malgré plusieurs analyses qui démontrent une certaines fréquence des notes du traducteur dans les textes littéraires ${ }^{6}$, leur apparition dans ces textes n'est pas généralement recommandée, car elles rompent la linéarité de la lecture et «fatiguent» le lecteur. En ce qui concerne les textes scientifiques, leur modèle par définition prévoit un nombre important tout d'abord de notes de l'auteur, de citations, de renvois bibliographiques, donc différentes formes de paratexte. Il devient alors aussi normal que dans ces textes les manifestations du traducteur complètent ce modèle. Kozłowska (2007 : 58) constate, par exemple, que la traduction du texte scientifique (plus particulièrement de sciences humaines) diffère, entre autres, de la traduction littéraire par le fait que la connaissance par le traducteur de la bibliographie de l'auteur de l'original est plus importante que celle du contexte culturel.

La classification des notes du traducteur a fait déjà l'objet de différentes analyses. Ne citons ici que Kozłowska (2007) et Kaufman (2009). Ce dernier, en intégrant les remarques de Kozłowska, a proposé une analyse de ce phénomène textuel en regroupant ces notes du point de vue de leur contenu, du point de vue de

\footnotetext{
${ }^{5}$ Cités dans la bibliographie du corpus.

${ }^{6}$ On peut consulter à ce propos quelques études réunies dans le volume sous la rédaction de Skibińska (2009).
} 
l'élément du texte auquel elles renvoient et du point de vue de leur fonction. L'analyse d'autres textes confirme ces observations. Ce qui par contre est plus intéressant de notre point de vue c'est leur fonction, c'est-à-dire, le degré de l'intervention du traducteur. Kaufman (2009 : 143) constate que de ce point de vue on peut distinguer des interventions du traducteur « médiatrices » découlant de son jugement du savoir des récepteurs et «co-créatrices » où le traducteur spécialiste complète le texte ou même entre en polémique avec l'auteur, par exemple ${ }^{7}$ :

\begin{tabular}{|l|l|}
\hline (...): l'enfant devient capable de trouver des & $(\ldots)$ : dziecko staje się zdolne do znajdowania no- \\
moyens nouveaux non plus seulement par & wych środków nie tylko po omacku, przez próby \\
tâtonnements extérieurs ou matériels, mais par & zewnętrzne lub materialne [7], ale drogą kombi- \\
combinaisons intériorisées qui aboutissent à une & nacji zinterioryzowanych, które doprowadzają do \\
compréhension soudaine ou insight. & nagłego zrozumienia, czyli insight. \\
& [7] Przez „,czynności materialne" Piaget rozumie \\
& czynności ruchowe oraz czynności praktyczne, \\
& wykonywane przez podmiot na przedmiotach, tzn. \\
& czynności ,zewnętrzne” w odróżnieniu od „we- \\
& wnętrznych”, czyli psychicznych. (Patrz przypis \\
& prof. A. Szemińskiej w: J. Piaget - Równoważenie \\
& struktur poznawczych, s. 27). (Przyp. tłumacza)
\end{tabular}

Ce type d'interventions du traducteur constitue donc presque une obligation pendant la traduction des textes scientifiques faisant partie des textes spécialisés. Pourtant, cette constatation n'est pas vraie pour tous les textes où se manifestent les langues de spécialité. Il faut bien distinguer les discours scientifiques liés à des domaines tels que la psychologie, la linguistique ou la physique, de vulgarisation (manuels, presse spécialisée) et des discours utilitaires (modes d'emploi, bulletin météo, contrat de bail). Non seulement les récepteurs de ces textes ne sont pas les mêmes, mais aussi leurs fonctions sont divergentes. Il en résulte, que la présence du traducteur dans les deux dernières catégories se manifeste rarement dans les paratextes, c'est pourquoi en comparant dans la suite la traduction d'un texte scientifique en psychologie de Piaget avec la traduction d'un numéro de la Revue Technique Automobile, , nous voulons nous concentrer sur les traces du traducteur intégrées dans le texte.

\subsection{TRACES DANS LE CORPS DU TEXTE}

Ces traces sont difficiles à voir sans la comparaison avec l'original. Elles sont présentes dans tous types de traduction. Souvent elles prennent forme d'un

\footnotetext{
${ }^{7}$ Cet exemple et les autres cités plus bas proviennent de deux corpus analysés par nos deux étudiantes de maîtrise : Kuźba (2015) et Barczewska (2014) (voir la bibliographie).

${ }^{8}$ L'analyse de ce corpus a été effectuée par Barczewska (2014). Cf. la bibliographie.
} 
certain développement définitionnel, d'une explicitation ou d'un ajout et se rapportent à des éléments jugés comme non transférables directement ou intraduisibles ${ }^{9}$.

\subsubsection{DÉFINITION DU RÉCEPTEUR}

Un texte scientifique s'adresse principalement à des spécialistes, scientifiques ou étudiants et les destinataires de sa traduction appartiennent au même groupe social. Par contre, un texte pragmatique vise des récepteurs plus variés. En plus, le public d'arrivée peut être défini d'une autre manière que le récepteur indigène. C'est par exemple le cas du corpus analysé (Revue Automobile):

\begin{tabular}{|l|l|}
\hline $\begin{array}{l}\text { Cet ouvrage est destiné aux professionnels de la } \\
\text { réparation ainsi qu'aux amateurs compétents. }\end{array}$ & $\begin{array}{l}\text { Odbiorcy: użytkownicy opisanych modeli samo- } \\
\text { chodów, pracownicy zaplecza technicznego moto- } \\
\text { ryzacji oraz wszyscy zainteresowani tymi samo- } \\
\text { chodami. (...) }\end{array}$ \\
$\begin{array}{l}\text { C'est donc volontairement que certaines infor- } \\
\text { Tłumacz i wydawca informują, że podjęli wszel- } \\
\text { mations - qui se déduisent naturellement de la } \\
\text { lecture du texte ou de l'examen d'un dessin - ne } \\
\text { sont pas davantage détaillées. }\end{array}$ & $\begin{array}{l}\text { kanych oraz porad zawartych w tej książce, (...) } \\
\text { danch }\end{array}$ \\
\hline
\end{tabular}

Dès le début on précise alors que les récepteurs de l'original sont des personnes plus compétentes que les récepteurs cible. On dit ouvertement que dans l'original certaines informations ont un caractère implicite. Par contre, dans la version $\mathrm{B}$, le traducteur informe qu'il a adopté le texte aux besoins des nouveaux récepteurs. Ces adaptations se font justement à l'intérieur du texte et non pas sous forme de paratextes. Si nous comparons cette traduction avec la traduction d'un texte scientifique (Piaget, Inhelder, 1999) ${ }^{10}$, nous constatons que la présence du traducteur dans le corps de ce texte est aussi très importante, malgré l'existence de ses interventions aussi dans les notes.

\subsubsection{TECHNIQUES EXPLICATIVES}

Les statistiques concernant un seul texte ne sont peut-être pas représentatives pour la totalité des traductions scientifiques, mais au moins donnent-elles une idée de l'ampleur du problème. La traduction du texte en question (Piaget,

\footnotetext{
${ }^{9}$ Nous avons discuté le problème de l'intraduisibilité et de ses solutions possibles dans : Tomaszkiewicz (2001, 2003, 2009, 2010).

${ }^{10}$ L'analyse de Kuźba (2015).
} 
Inhelder, 1999) compte 116 pages où apparaissent 331 exemples d'intervention $\mathrm{du}$ traducteur sous forme de différentes techniques explicatives. Proportionnellement, les interventions du traducteur dans le corps du texte constituent $83 \%$ de tous les exemples et seulement $17 \%$ sont des explications dans les notes.

En ce qui concerne la traduction de la revue spécialisée (Revue Technique Automobile, 2002) qui d'ailleurs est destinée à un autre type de récepteur que l'original, toutes les explications supplémentaires sont contenues dans le texte principal. Elles constituent le plus souvent un ajout qui «consiste à l'ajout d'informations inexistantes sur l'original par le biais d'une explication ou d'une expansion» (Guidère, 2010: 86). Ces expansions peuvent contenir des informations inexistantes dans l'original sous forme de phrases entières, d'explicitation des implicites ou de précisions terminologiques présentées en registre commun (standard). Regardons à titre d'exemple :

\begin{tabular}{|l|l|}
\hline $\begin{array}{l}\text { 1. Pour faciliter le déverrouillage, manœuv- } \\
\text { rer le volant à droite et à gauche en } \\
\text { tournant la clé. }\end{array}$ & $\begin{array}{l}\text { W celu ułatwienia odblokowania kierownicy zaleca się } \\
\text { obracanie kierownicą o niewielki kąt w prawo i w lewo } \\
\text { przy jednoczesnym obracaniu kluczyka w prawo. Zablo- } \\
\text { kowanie kierownicy następuje po wyjęciu kluczyka } \\
\text { i obróceniu jej (w dowolnym kierunku) aż do usłysze- } \\
\text { nia charakterystycznego trzasku zapadki blokady. }\end{array}$ \\
\hline $\begin{array}{l}\text { 2. Les seuils de changement de rapport } \\
\text { (montée de rapport et rétrograde) dé- } \\
\text { pendent du mode sélectionné et de la } \\
\text { conduite du conducteur (fonctionne- } \\
\text { ment autoadaptatif). }\end{array}$ & $\begin{array}{l}\text { Prędkość zmiany przełożeń (w górę i w dół) zależą od wy- } \\
\text { branego programu jazdy (normalna, zimowa, sportowa) } \\
\text { i sposobu prowadzenia samochodu przez kierowcę (dzia- } \\
\text { łanie samoadaptacyjne). }\end{array}$ \\
\hline $\begin{array}{l}\text { 3. Le contacteur à clé est situé à droite sur } \\
\text { le bloc de la colonne de direction. }\end{array}$ & $\begin{array}{l}\text { Wyłącznik zapłonu (tzw. stacyjka) znajduje się po prawej } \\
\text { stronie kolumny kierownicy. }\end{array}$ \\
\hline $\begin{array}{l}\text { 4. Poussoirs cylindriques avec dispositif } \\
\text { de rattrapage hydraulique de jeu (...) }\end{array}$ & $\begin{array}{l}\text { Zastosowano walcowe popychacze (tzw. szklankowe) za- } \\
\text { wierające urządzenie do hydraulicznego kasowania luzu (...) }\end{array}$ \\
\hline
\end{tabular}

Le premier exemple montre que le traducteur a introduit toute une phrase inexistante dans l'original pour adapter le texte au besoins du récepteur cible, en lui expliquant une manœuvre contraire au déverrouillage. Dans le deuxième il a explicité ce qui pourrait être compris implicitement par un récepteur de départ, jugé comme plus compétent que le récepteur cible (voir le fragment de l'introduction). Enfin, dans les exemples 3 et 4 on a reformulé deux termes de spécialité en lexèmes appartenant au langage standard.

En ce qui concerne les interventions du traducteur du texte scientifique, elles sont relativement nombreuses, c'est pourquoi nous ne citons que quelques exemples illustratifs. La technique la plus fréquente c'est l'explicitation de l'implicite. Aussi bien Kozłowska (1995: 39) que Wawrzyniak (1991 : 97) observent que très fréquemment les originaux sont plus concis que la traduction. 
On a l'impression que les traducteurs essayent de formuler le texte d'arrivée d'une manière qui doit faciliter la lecture au niveau terminologique et syntaxique.

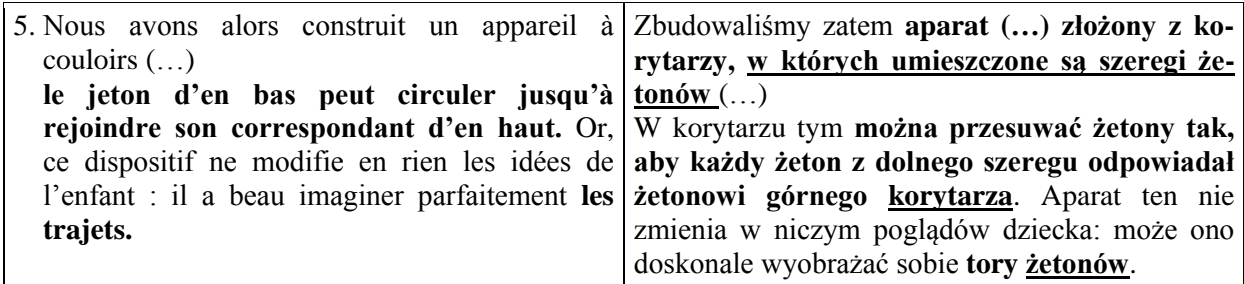

Nous voyons tout de suite combien d'éléments présentés dans l'original implicitement sont explicités dans la traduction. Ces explicitations concernent souvent certains termes qui au lieu d'être repris par une reformulation, sont répétées plusieurs fois.

Le même souci de précision terminologique dicte au traducteur la reprise d'un terme par un équivalent dans une autre langue, par exemple :

\begin{tabular}{|l|l|}
\hline 6. Opossum (sorte de fossile vivant) & Oposy (Didelphis marsupialis) \\
\hline 7. La théorie de la Gestalt & Teoria postaci (Gestalt) \\
\hline
\end{tabular}

Un autre phénomène qui apparaît fréquemment dans la traduction c'est la renonciation à différents types de relations anaphoriques. Dans beaucoup de passages où dans l'original un terme est repris plus loin par une anaphore grammaticale sous forme pronominale, dans la traduction il est répété dans sa forme originale.

\begin{tabular}{|l|l|}
\hline $\begin{array}{l}\text { 8. La perception constitue }(\ldots) \text { un cas particulier } \\
\text { des activités sensori-motrices. Mais son ca- } \\
\text { ractère particulier consiste en ceci qu'elle relève } \\
\text { de l'aspect figuratif de la connaissance du réel } \\
\text { (...). }\end{array}$ & $\begin{array}{l}\text { Percepcja stanowi (...) szczególny przypadek } \\
\text { działan zmysłowo-ruchowych. Ta szczególnośćc } \\
\text { polega na tym, że percepcja wypływa z figu- } \\
\text { ralnego aspektu wiedzy o rzeczywistości }(\ldots)\end{array}$ \\
\hline $\begin{array}{l}\text { 9. (..) la région des lobes occipitaux qui reçois } \\
\text { une grande proportion de fibres }(\ldots) \text { est moins } \\
\text { développée }(\ldots) \text { que les régions recevant les } \\
\text { leurs de la périphérie de la rétine. }\end{array}$ & $\begin{array}{l}(\ldots) \text { rejon płatów potylicznych, do których do- } \\
\text { ciera większa część włókienek nerwowych }(\ldots) \\
\text { jo których docierają wókienka od peryferii siat- } \\
\text { kówki. }\end{array}$ \\
\hline
\end{tabular}


Les exemples de ce type sont abondants et résultent probablement du soucis d'augmenter la présence de la terminologie spécialisée dans le textes scientifique. Le même souci amène le traducteur à renoncer à la reformulation d'un terme par un autre lexème et à le reprendre tel quel.

10. l'escargot tout en avançant peut rester immobile par rapport au point extérieur parce que le mouvement de la planche compense sans l'annuler celui de l'animal. ślimak posuwając się stale naprzód może nie zmienić położenia w stosunku do punktu zewnętrznego, ponieważ ruch deseczki kompensuje ruch ślimaka, nie anulując go.

Toutes ces interventions du traducteur montrent sa présence dans le texte en lui attribuant parfois le rôle appelé par Kaufman (cf. plus haut 3.1.) de « cocréateur ». Dans beaucoup de cas analysés les changements introduits par le traducteur n'étaient nécessaires ni du point de vue linguistique, ni stylistique. C'est donc lui qui a pris ces décisions en voulant assurer la meilleure compréhension du récepteur. Pourtant, cette volonté d'assurer une plus grande clarté peut causer un effet de surtraduction, dans certains cas jugée comme une faute de traduction. «Faute de traduction qui consiste à traduire explicitement des éléments du texte de départ qui devraient rester implicites dans le texte d'arrivée » (Delisle et al., 1999).

11. Mais la question est de savoir si le bébé Pytanie tylko, czy u dziecka obserwujemy „efekt présente ou non les effets "écran " et zasłony” $\mathbf{i}$ „efekt tunelu” zanim powstała u niego «tunnel» avant d'avoir construit la per- stałość przedmiotu. Doświadczenie przeczy takiemanence de l'objet. Or, pour le second, mu przypuszczeniu, jeśli chodzi o „efekt tunelu”. l'expérience montre qu'il n'en est rien.

L'analyse de ce fragment et de plusieurs autres montre qu'on aurait très bien pu sauvegarder la forme de l'original, sans abuser de répétitions, et de reprises multiples de termes. Cette observation rejoint l'opinion de Hrehorowicz :

Il (le traducteur) pourra remarquer facilement que le texte d'arrivée est toujours plus long que le texte de départ. Ce phénomène est bien connu des traductologues qui l'expliquent par une sorte d'excès de zèle : le traducteur juge inconsciemment ses destinataires moins perspicaces qu'ils ne le sont et tend à expliciter et à expliquer (Hrehorowicz, 1993 : XIII).

\section{CONCLUSION}

Le but de cet article consistait avant tout à démontrer que dans la traduction des textes spécialisés de la même manière que dans la traduction d'autres types de textes, textes littéraires y compris, la présence du traducteur se manifeste 
d'une manière plus ou moins explicite. Cette présence est à récupérer soit dans le paratexte soit dans le corps du texte lui-même. Comme la présence de différentes manifestations du paratexte dans un texte scientifique constitue une norme rédactionnelle de ce genre, la présence de notes du traducteur ou de certaines informations dans les préfaces participe à la définition de ce modèle de texte. Par contre, en ce qui concerne un texte pragmatique, sa caractéristique ne prévoit pas de renvois bibliographiques, définitions, traduction des exemples. Le but d'un texte pragmatique consiste à donner à son usager des conseils pratiques. Le récepteur de ce texte n'est pas obligé d'être un spécialiste, mais il attend des explications, des descriptions, des mises au point. Ces constatations dévoilent la différence majeure entre les stratégies d'un traducteur d'un texte scientifique et d'un texte pragmatique. Dans le premier cas il est obligé de reconstruire le style scientifique avec les renvois, les explications, les discussions terminologiques, les relations inter discursives. Dans le deuxième, en principe il doit aider un non spécialiste à résoudre ses problèmes particuliers.

Il en résulte que la présence du traducteur dans le corps de ces genres se manifeste différemment. Les techniques utilisées dans la traduction d'un texte scientifique les plus fréquentes sont: l'explicitation de l'implicite, la concrétisation du sens, les précisions terminologiques, ce qui peut, par endroits, mener à des répétitions abusives. Dans la traduction d'un texte utilitaire on va le plus souvent se servir de développements définitionnels, d'ajouts, d'introduction d'un jargon où les termes spécialisés peuvent être remplacés par le vocabulaire courant. Or, la présence de ces techniques montre sans équivoque le rôle actif du traducteur de textes spécialisés comme médiateur mais aussi comme co-créateur. 


\section{BIBLIOGRAPHIE}

Ballard, M. (1995). De Ciceron à Benjamin. Traducteurs, Traductions, Réflexions. Lille : Presses Universitaires de Lille.

Barańczak, S. (1992). Ocalone w tlumaczeniu. Poznań : Wydawnictwo a5.

Barczewska, A. (2014). La traduction des textes techniques sur l'exemple de la "Revue Technique Automobile ».Travail de maîtrise préparé sous la direction de Teresa Tomaszkiewicz, à l'Université Adam Mickiewicz à Poznań.

Bastin, G. L. et Cormier, M. (2007). Profession traducteur. Montréal : Les Presses de l'Université de Montréal.

Cabré, M.T. (1998). La terminologie. Théorie, méthodes, applications (trad. du catalan). Ottawa : Les Presses de l'Université d'Ottawa.

Charnock, R. (1999). «Les langues de spécialité et le langage technique: considérations didactiques », $A S p$ [En ligne], 23-26 | 1999, mis en ligne le 09 novembre 2011, consulté le 26 juin 2015. URL : http://asp.revues.org/2566; DOI: 10.4000/asp.2566, pp. 281-302.

Dąmbska-Prokop, U. (1997). Śladami tlumacza. Szkice. Kraków : Edukator-Viridis.

Dąmbska-Prokop, U. (2000). Mała encyklopedia przekładoznawstwa. Częstochowa : Edukator.

Delisle, J., Lee-Jahnke, H. \& Cormier, M.C. (1999). Terminologie de la traduction. Amsterdam Philadelphia : John Benjamins Publishing Company.

Frandsen, F. (1998). «Langue générale et langue de spécialité - une distinction asymétrique ?» In Y. Gambier (Éd.), Discours professionnels en français, Frankfurt/M, Peter Lang, pp.15-34.

Guidère, M. (2010). Introduction à la traductologie : penser la traduction: hier, aujourd'hui, demain. Bruxelles : De Boeck.

Hrehorowicz, U. (1993). Savoir trahir. Kraków : C\&D, p. XIII.

Kaufman, S. (2009). „Przypisy tłumacza tekstu specjalistycznego: od mediacji do współuczestnictwa”. In E. Skibińska (Éd.), Przypisy ttumacza. Wrocław - Kraków : Księgarnia Akademicka.

Kocourek, R. (1991). La langue française de la technique et de la science. Vers une linguistique de la langue savante. Wiesbaden : Branstetter.

Kozłowska, Z. (1995). O przekładzie tekstu naukowego (na materiale tekstów językoznawczych). Warszawa : Wydawnictwa Uniwersytetu Warszawskiego.

Kuźba, D. (2015). Techniques explicatives dans la traduction du texte scientifique sur l'exemple de „La psychologie de l'enfant” de J. Piaget et B. Inhelder. Travail de maîtrise préparé sous la direction de Teresa Tomaszkiewicz, à l'Université Adam Mickiewicz à Poznań.

Lerat, P. (1995). Les langues spécialisées. Paris : Presses Universitaires de France.

Lerat, P. (1997). « Approches linguistiques des langues spécialisées ». Asp, 15-18, 1-10.

Lüdi, G. (1987). «Aspects énonciatifs de la traduction », Cahiers du Département des Langues et Sciences du Langage 5, 53-71.

Mortureux, M.-F. (1995). "Les vocabulaires scientifiques et techniques ». In J.-Cl. Béacco \& S. Moirand (Éd.), Les enjeux des discours spécialisés. Cediscor/Paris 3, pp.13-26.

Quemada, B. (1978). « Technique et langage ». In B. Gille (Éd.), Histoire des techniques. Paris : Gallimard, pp. 1146-1240.

Sager, J. C., Dungworth, D., McDonald, P. F. (1980). English special languages. Principles and practice in Science and Technology. Wiesbaden : Brandstetter.

Skibińska, E. (2009). (Éd.), Przypisy ttumacza. Wrocław - Kraków : Księgarnia Akademicka.

Tomaszkiewicz, T. (2001). "Transfert des références culturelles dans les sous-titres filmiques». In Y. Gambier \& H. Gottlieb (Éds.), (Multi)Media Translation. Amsterdam - Philadelphia : John Benjamins Publishing Company, pp. 237-249. 
Tomaszkiewicz, T. (2003). " Traduction des références culturelles dans les films sous-titrés ». In H.-J. Lüsebrink \& K. Walter (Éd.), Interkulturelle Medienanalyse. St. Ingbert : Röhrig Universitätsverlag, 2003, pp. 190-211.

Tomaszkiewicz, T. (2009). „Granice przekładalności współczesnej literatury akadyjskiej w świetle myśli Olgierda Wojtasiewicza". In K. Hejwowski, A. Szczęsny \& U. Topczewska, 50 lat polskiej translatoryki. Warszawa: ILS, pp.163-174.

Tomaszkiewicz, T. (2010). „Areas of Untranslatability in Audiovisual Transfers”. In Ł. Bogucki \& K. Kredens (Éd.), Perspectives on Audiovisual Translation, Frankfurt/M, Berlin - Bern Bruxells - New York - Oxford - Wien : Peter Lang, pp. 93-106.

Varantola, K. (1986). "Special Language and General Language: Linguistic and Didactic Aspects », ALSED-LSP Newsletter, vol. 9, 2(23), 10-19.

Walkiewicz, B. (2013). O architekturze tekstów o architekturze w perspektywie przektadu. Poznań : Wydawnictwo Naukowe UAM.

Wawrzyniak, Z. (1991). Praktyczne aspekty translacji literackiej na przykładzie języków niemieckiego i angielskiego. Warszawa : PWN.

\section{BIBLIOGRAPHIE DU CORPUS}

Piaget, J. \& Inhelder, B. (1993). La psychologie de l'enfant. Paris : Presses Universitaires de France.

Piaget, J. \& Inhelder, B. (1999). Psychologia dziecka. Wrocław : Siedmiogród.

Revue Technique Automobile (2002) exclusivement réservé aux Citröen Xsara essence Tu 1.4. et 1.6. 16V, publié par ETAI, No 646, et sa traduction polonaise. 
